did not have a history of cardiovascular disease. Among the 765 people who began taking bloodpressure-lowering drugs during the follow-up period (mean duration 7.1 years), increasing baseline urinary albumin level was associated with decreasing risk of cardiovascular morbidity or mortality relative to individuals who did not start taking blood-pressure-lowering agents (hazard ratios $0.87,0.58$, and 0.37 for individuals with urinary albumin excretion of $<15 \mathrm{mg} /$ day, $\geq 15 \mathrm{mg} /$ day, and $\geq 30 \mathrm{mg} /$ day, respectively). Furthermore, people with elevated baseline urinary albumin levels who were treated with only angiotensin-converting-enzyme inhibitors or angiotensin-receptor blockers had a lower risk of future cardiovascular events than those who were treated with only other agents (hazard ratio 0.63).

The authors note that albuminuria reflects endothelial damage due to atherosclerosis; therefore, blood-pressure-lowering drugs might help to prevent cardiovascular events in individuals with albuminuria by inhibiting atherosclerosis.

Original article Boersma C et al. (2008) Baseline

albuminuria predicts the efficacy of blood pressure-

lowering drugs in preventing cardiovascular events. Br J Clin Pharmacol 65: 723-732

\section{Diuretic versus CCB as second- line therapy in diabetic patients with hypertension}

Adding a calcium-channel blocker (CCB) or a diuretic to an angiotensin-converting enzyme inhibitor (ACEl) further reduces blood pressure in patients who have diabetes and hypertension; however, the impact on albuminuria is unknown.

The 52-week double-blind, randomized, controlled GUARD (Gauging Albuminuria Reduction with Lotrel in Diabetic Patients With Hypertension) trial enrolled 332 hypertensive patients with albuminuria and type 2 diabetes mellitus to test the hypothesis that the combination of the ACEI benazepril and the CCB amlodipine would produce comparable reductions in blood pressure and albuminuria to those elicited by the combination of benazepril and the diuretic hydrochlorothiazide.

Both combinations significantly reduced blood pressure and urinary albumin to creatinine ratio $(P<0.0001$ for all); however, the median reduction in urinary albumin to creatinine ratio achieved with the benazepril-diuretic combination was significantly greater than that obtained with the benazepril-CCB combination (-72.1\% vs $-40.5 \%$; $P<0.0001)$. Furthermore, the percentage of patients with microalbuminuria who attained a normal urinary albumin to creatinine ratio was higher with combined $\mathrm{ACEI}$-diuretic therapy than with combined ACEI-CCB treatment $(69.2 \%$ vs $47.8 \% ; P=0.0004)$. Conversely, the mean reduction in diastolic blood pressure was significantly greater in the ACEI-CCB group than in the ACEI-diuretic group $(-13.1 \mathrm{mmHg}$ vs $-9.97 \mathrm{mmHg} ; P=0.02)$. Both combinations were well tolerated.

The authors note that there was a greater decline in estimated glomerular filtration rate among the patients who received the diuretic rather than the $\mathrm{CCB}$, which could account for the larger decrease in albuminuria in the diuretic group.

Original article Bakris GL et al. (2008) Effects of different ACE inhibitor combinations on albuminuria: results of the GUARD study. Kidney Int 73: 1303-1309

\section{ACE gene I/D polymorphism influences losartan responsive- ness in diabetic nephropathy}

An insertion (I)/deletion (D) polymorphism in the angiotensin-converting-enzyme (ACE) gene is known to influence the risk of patients with diabetes developing nephropathy. Parving et al. assessed the impact of the ACE I/D polymorphism on outcomes and the response to angiotensin-II-receptor blockade in patients with type 2 diabetes and nephropathy.

The authors performed a prespecified analysis of 1,435 patients who had participated in the randomized, double-blind, placebocontrolled RENAAL study and had known ACE I/D genotype. The mean follow-up period was 3.4 years. Within the placebo arm of the study, individuals with either the ID or DD genotype were, respectively, $17.5 \%$ and $38.1 \%$ more likely than individuals with the II genotype to experience the composite primary end point of doubling of baseline serum creatinine concentration, development of end-stage renal disease, or death $(P=0.029)$. In the losartan arm of the study, however, the effect of genotype on outcome was not significant. Compared with the placebo group, the risk of reaching the 